Bull. Korean Math. Soc. 52 (2015), No. 4, pp. 1059-1068

http://dx.doi.org/10.4134/BKMS.2015.52.4.1059

\title{
EXISTENCE AND LARGE TIME BEHAVIOR OF SOLUTIONS TO A FOURTH-ORDER DEGENERATE PARABOLIC EQUATION
}

\author{
Bo Liang, Meishan Wang, and Ying Wang
}

\begin{abstract}
The paper is devoted to studying a fourth-order degenerate parabolic equation, which arises in fluid, phase transformation and biology. Based on the existence and uniqueness of one semi-discrete problem, two types of approximate solutions are introduced. By establishing some necessary uniform estimates for those approximate solutions, the existence and uniqueness of the corresponding parabolic problem are obtained. Moreover, the long time asymptotic behavior is established by the entropy functional method.
\end{abstract}

\section{Introduction}

In the paper, we mainly study the following fourth-order degenerate parabolic problem:

$$
\begin{aligned}
& u_{t}+\Delta\left(|\Delta u|^{p-2} \Delta u\right)-\nabla \cdot\left(f(x)|\nabla u|^{q-2} \nabla u\right)=g(x) \text { in } Q_{T}, \\
& u=\Delta u=0 \text { on } \Gamma, \\
& u(x, 0)=u_{0}(x) \text { on } \Omega,
\end{aligned}
$$

where $\Omega \subset \mathbb{R}^{N}$ is a bounded domain, $\partial \Omega$ is smooth enough, $Q_{T}=\Omega \times(0, T)$, $\Gamma=\partial \Omega \times(0, T)$ and $p, q>1$. The equation (1) can be taken as the generalized Cahn-Hilliard equation or thin film equation. Particularly, if $p=2$, it becomes the Cahn-Hilliard model, which appears in the description of phase transformation theory (see [3]). By replacing $|\Delta u|^{p-2}$ with $u^{m}(m>0)$, the equation (1) is just the thin film equation, which can be used to study the motion of a very thin layer of viscous in compressible fluids along an inclined plane. The unknown function $u$ represents the thickness of the film (see [8]).

Received March 30, 2013; Revised April 2, 2015

2010 Mathematics Subject Classification. Primary 35G20, 35G30, 35G61, 35Q35.

Key words and phrases. fourth-order parabolic, fourth-order elliptic, Cahn-Hilliard equation, thin film equation, long time behavior.

This work was financially supported by the National Natural Science Foundation of China 11201045. 
Mathematically, there have been some research results about the fourthorder degenerate parabolic equations. For the one dimensional thin film equation, Bernis, Friedman [2] and Grün [6] have obtained the existence of a class of weak solutions from some energy estimates. Moreover, Carrillo and Toscani addressed the long time behavior of solutions in [4] by the entropy functional method. In addition, Xu and Zhou (see [10] and [11]) have studied the nonlinear fourth-order equation $u_{t}+\nabla \cdot\left(|\nabla \Delta u|^{p-2} \nabla \Delta u\right)=f$ and they got the existence and stability of weak solutions.

It is pointed out that the comparison principle does not hold for the fourthorder partial differential equations and so we have to rely on the energy estimate method to obtain the existence of solutions.

The weak solution of (1)-(3) is defined as follows.

Definition. A function $u$ is said to be a weak solution of the problem (1)-(3) if the following conditions hold:

1. $u \in C\left([0, T] ; L^{p}(\Omega)\right) \cap L^{p}(0, T ; \mathcal{H}), \frac{\partial u}{\partial t} \in L^{p^{\prime}}\left(0, T ; \mathcal{H}^{\prime}\right), u(x, 0)=u_{0}(x)$ in $\Omega$;

2. For each $\varphi \in L^{p}(0, T ; \mathcal{H})$, it has

$$
\begin{aligned}
& \int_{0}^{T}\left\langle\frac{\partial u}{\partial t}, \varphi\right\rangle \mathrm{d} t+\iint_{Q_{T}}|\Delta u|^{p-2} \Delta u \Delta \varphi \mathrm{d} x \mathrm{~d} t \\
& +\iint_{Q_{T}} f(x)|\nabla u|^{q-2} \nabla u \nabla \varphi \mathrm{d} x \mathrm{~d} t=\iint_{Q_{T}} g(x) \varphi \mathrm{d} x \mathrm{~d} t
\end{aligned}
$$

where we have used the following notations:

$$
\begin{aligned}
& \mathcal{H} \doteq W_{0}^{1, p}(\Omega) \cap W^{2, p}(\Omega), \\
& \mathcal{H}^{\prime} \doteq\left(W_{0}^{1, p}(\Omega) \cap W^{2, p}(\Omega)\right)^{\prime}, \\
& p^{\prime} \doteq \frac{p}{p-1}\left(\text { i.e., } \frac{1}{p^{\prime}}+\frac{1}{p}=1\right),
\end{aligned}
$$

and $\langle\cdot, \cdot\rangle$ denotes the inner product between $\mathcal{H}^{\prime}$ and $\mathcal{H}$.

The main results of the paper including the existence and long time behavior are as follows.

Theorem 1.1. Assume $u_{0} \in L^{p}(\Omega) \cap L^{2}(\Omega), f \in L^{\infty}(\Omega), f \geq 0, g \in L^{p^{\prime}}(\Omega)$ and $q \leq \frac{N p}{N-p}$ if $p<N$. Then the problem (1)-(3) admits a unique weak solution.

Theorem 1.2. Let $u$ be a weak solution of (1)-(3). Assume $\left\|u_{0}\right\|_{L^{2}(\Omega)}>0$, $f \in L^{\infty}(\Omega), f \geq 0$ and $g \equiv 0$. Then it has

$$
\|u\|_{L^{2}(\Omega)}(t) \leq \begin{cases}\left(\left\|u_{0}\right\|_{L^{2}(\Omega)}^{2-p}+C\left(\frac{p}{2}-1\right) t\right)^{-\frac{1}{p-2}}, & p>2 \\ \left\|u_{0}\right\|_{L^{2}(\Omega)} e^{-C t}, & p=2 .\end{cases}
$$

For $p<2$, there exists a time constant $\bar{t}>0$ such that $u=0$ for all $t>\bar{t}$. 
Throughout the paper, $C$ is denoted as a general positive constant, and may change from line to line. The paper is arranged as follows. We will prove the existence and uniqueness of solutions in Section 2. The long time asymptotic behavior will be established in Section 3 .

\section{Existence and uniqueness}

In order to prove Theorem 1.1, let us define a semi-discrete problem of (1)(3) at first:

(4) $\frac{1}{h}\left(u_{k}-u_{k-1}\right)+\Delta\left(\left|\Delta u_{k}\right|^{p-2} \Delta u_{k}\right)-\nabla \cdot\left(f(x)\left|\nabla u_{k}\right|^{q-2} \nabla u_{k}\right)=g(x)$ in $\Omega$,

(5) $u_{k}=\Delta u_{k}=0 \quad$ on $\partial \Omega$,

for each positive integer $n, u_{k}=u(x, k h), h=\frac{T}{n}$ and $k=1,2, \ldots, n$. To gain its existence, we need introduce a functional of $u_{k}$ :

$$
\begin{aligned}
\mathcal{M}\left[u_{k}\right]= & \frac{1}{2 h} \int_{\Omega}\left|u_{k}\right|^{2} \mathrm{~d} x+\frac{1}{p} \int_{\Omega}\left|\Delta u_{k}\right|^{p} \mathrm{~d} x \\
& +\frac{1}{q} \int_{\Omega} f(x)\left|\nabla u_{k}\right|^{q} \mathrm{~d} x+\int_{\Omega}\left(g(x)+\frac{1}{h} u_{k-1}\right) u_{k} \mathrm{~d} x .
\end{aligned}
$$

Next we employ (6) to establish:

Lemma 2.1. The problem (4)-(5) admits a unique weak solution $u_{k} \in \mathcal{H}$ satisfying

$$
\begin{aligned}
& \frac{1}{h} \int_{\Omega}\left(u_{k}-u_{k-1}\right) \psi \mathrm{d} x+\int_{\Omega}\left|\Delta u_{k}\right|^{p-2} \Delta u_{k} \Delta \psi \mathrm{d} x \\
& +\int_{\Omega} f(x)\left|\nabla u_{k}\right|^{q-2} \nabla u_{k} \nabla \psi \mathrm{d} x=\int_{\Omega} g(x) \psi \mathrm{d} x, \\
& \frac{1}{h} \int_{\Omega}\left|u_{k}\right|^{2} \mathrm{~d} x+\int_{\Omega}\left|\Delta u_{k}\right|^{p} \mathrm{~d} x \leq \frac{1}{h} \int_{\Omega}\left|u_{k-1}\right|^{2} \mathrm{~d} x+C, \\
& \int_{\Omega}\left|u_{k}\right|^{2} \mathrm{~d} x+h \sum_{i=1}^{k} \int_{\Omega}\left|\Delta u_{i}\right|^{p} \mathrm{~d} x \leq \int_{\Omega}\left|u_{0}\right|^{2} \mathrm{~d} x+C T,
\end{aligned}
$$

for $k=1,2, \ldots, n$ and $\psi \in \mathcal{H}$.

Proof. By applying Young's inequality, Hölder's inequality, the Sobolev embedding theorem (see [1]) and $L^{p}$-estimate (see [5]), we have

$$
\begin{aligned}
\left|\int_{\Omega}\left(g(x)+\frac{1}{h} u_{k-1}\right) u_{k} \mathrm{~d} x\right| & \leq\left\|h+\frac{1}{h} u_{k-1}\right\|_{L^{p^{\prime}}(\Omega)}\left\|u_{k}\right\|_{L^{p}(\Omega)} \\
& \leq \frac{1}{2 p}\left\|\Delta u_{k}\right\|_{L^{p}(\Omega)}^{p}+C\left\|h+\frac{1}{h} u_{k-1}\right\|_{L^{p^{\prime}}(\Omega)}^{p^{\prime}} .
\end{aligned}
$$


Hence, according to (6) and (10), we get

$$
\begin{aligned}
\mathcal{M}\left[u_{k}\right] & \geq \frac{1}{2 h} \int_{\Omega}\left|u_{k}\right|^{2} \mathrm{~d} x+\frac{1}{2 p} \int_{\Omega}\left|\Delta u_{k}\right|^{p} \mathrm{~d} x+\frac{1}{q} \int_{\Omega} f(x)\left|\nabla u_{k}\right|^{q} \mathrm{~d} x-C \\
& \geq-C
\end{aligned}
$$

which can show that the functional $\mathcal{M}\left[u_{k}\right]$ is bounded:

$$
-C \leq \inf _{v \in \mathcal{H}} \mathcal{M}[v] \leq \mathcal{M}[0]=0
$$

Furthermore, there exists a minimizing sequence $\left\{u_{k}^{l}\right\}_{k=1}^{\infty} \subset \mathcal{H}$ satisfying

$$
\mathcal{M}\left[u_{k}^{l}\right] \rightarrow \inf _{v \in \mathcal{H}} \mathcal{M}[v]
$$

when $l \rightarrow+\infty$, and then the $L^{p}$-estimate of the second-order elliptic equation yields

$$
\left\|u_{k}^{l}\right\|_{W^{2, p}(\Omega)} \leq C\left\|\Delta u_{k}^{l}\right\|_{L^{p}(\Omega)} .
$$

Therefore, there exist a subsequence of $\left\{u_{k}^{l}\right\}$ and a function $u_{k} \in \mathcal{H}$ such that $u_{k}^{l} \rightarrow u_{k}$ weakly in $\mathcal{H}$. For $p>1$, applying the weakly lower semi-continuity, we have

$$
\mathcal{M}[u] \leq \liminf _{l \rightarrow \infty} \mathcal{M}\left[u_{k}^{l}\right]=\inf _{v \in \mathcal{H}} \mathcal{M}[v],
$$

which implies $\mathcal{M}\left[u_{k}\right]=\inf _{v \in \mathcal{H}} \mathcal{M}[v]$. A standard proof guarantees that the function $u_{k}$ is a weak solution of (4)-(5).

We take $\psi=u_{k}$ as a test function in (7) to get

$$
\begin{aligned}
& \frac{1}{h} \int_{\Omega}\left|u_{k}\right|^{2} \mathrm{~d} x+\int_{\Omega}\left|\Delta u_{k}\right|^{p} \mathrm{~d} x+\int_{\Omega} f(x)\left|\nabla u_{k}\right|^{q} \mathrm{~d} x \\
\leq & \frac{1}{2 h} \int_{\Omega}\left|u_{k-1}\right|^{2} \mathrm{~d} x+\frac{1}{2 h} \int_{\Omega}\left|u_{k}\right|^{2} \mathrm{~d} x+\int_{\Omega} g(x) u_{k} \mathrm{~d} x .
\end{aligned}
$$

Similar to (10), we can prove that $\int_{\Omega} g(x) u_{k} \mathrm{~d} x \leq \frac{1}{2} \int_{\Omega}\left|\Delta u_{k}\right|^{p} \mathrm{~d} x+C$, and then we have (8). The inequality (9) can be obtained from (8) directly.

Finally, for given $u_{k-1}$, we will show the uniqueness. Let $w_{1}$ and $w_{2}$ be two solutions of (4)-(5), and take $w_{1}-w_{2}$ as a test function in the difference of the corresponding equations to get

$$
\begin{aligned}
& \frac{1}{h} \int_{\Omega}\left(w_{1}-w_{2}\right)^{2} \mathrm{~d} x \\
= & -\int_{\Omega}\left(\left|\Delta w_{1}\right|^{p-2} \Delta w_{1}-\left|\Delta w_{2}\right|^{p-2} \Delta w_{2}\right)\left(\Delta w_{1}-\Delta w_{2}\right) \mathrm{d} x \\
& -\int_{\Omega} f(x)\left(\left|\nabla w_{1}\right|^{q-2} \nabla w_{1}-\left|\nabla w_{2}\right|^{q-2} \nabla w_{2}\right)\left(\nabla w_{1}-\nabla w_{2}\right) \mathrm{d} x \\
\leq & 0,
\end{aligned}
$$

where we have used the fact $\left(|x|^{m-2} x-|y|^{m-2} y\right)(x-y) \geq 0$ for $m>1$ and $x, y \in \mathbb{R}\left(\right.$ or $\left.\mathbb{R}^{N}\right)$. Therefore, one has $w_{1}=w_{2}$ a.e. in $\Omega$. 
Fix a positive integer $n$, and define the first approximate solution as

$$
w^{(n)}(x, t)=\sum_{k=1}^{n} \chi_{k}(t) u_{k}(x)
$$

where

$$
\chi_{k}(t)= \begin{cases}1, & t \in((k-1) h, k h] \\ 0, & \text { elsewhere }\end{cases}
$$

for $k=1, \ldots, n$. We need some uniform estimates in $n$ as follows.

Lemma 2.2. It has

$$
\left\|w^{(n)}\right\|_{L^{\infty}\left(0, T ; L^{2}(\Omega)\right)}+\left\|w^{(n)}\right\|_{L^{p}(0, T ; \mathcal{H})} \leq C .
$$

Proof. For any $t \in(0, T)$, there exists a positive integer $k \in\{1, \ldots, n\}$ such that $t \in((k-1) h, k h]$, and then we have $\left\|w^{(n)}(x, t)\right\|_{L^{2}(\Omega)}^{2}=\left\|u_{k}(x)\right\|_{L^{2}(\Omega)}^{2} \leq C$. It gives $\left\|w^{(n)}\right\|_{L^{\infty}\left(0, T ; L^{2}(\Omega)\right)} \leq C$.

According to (9), we have $\iint_{Q_{T}}\left|\Delta w^{(n)}\right|^{p} \mathrm{~d} x \mathrm{~d} t=h \sum_{k=1}^{n} \int_{\Omega}\left|\Delta u_{k}\right|^{p} \mathrm{~d} x \leq C$. A simple argument completes the proof.

The second approximate solution is defined by

$$
u^{(n)}(x, t)=\sum_{k=1}^{n} \chi_{k}(t)\left[\lambda_{k}(t) u_{k}(x)+\left(1-\lambda_{k}(t)\right) u_{k-1}(x)\right]
$$

where

$$
\lambda_{k}(t)= \begin{cases}\frac{t}{h}-(k-1), & \text { if } t \in((k-1) h, k h] \\ 0, & \text { otherwise. }\end{cases}
$$

The following uniform estimates hold for $u^{(n)}$.

\section{Lemma 2.3. It has}

$$
\left\|\frac{\partial u^{(n)}}{\partial t}\right\|_{L^{p^{\prime}\left(0, T ; \mathcal{H}^{\prime}\right)}}+\left\|u^{(n)}\right\|_{L^{\infty}\left(0, T ; L^{2}(\Omega)\right)} \leq C .
$$

Proof. In view of (13), we have

$$
\frac{\partial u^{(n)}}{\partial t}=\frac{1}{h} \sum_{k=1}^{n} \chi_{k}\left(u_{k}-u_{k-1}\right)
$$

and then

$$
\begin{aligned}
\int_{0}^{T}\left\|\frac{\partial u^{(n)}}{\partial t}\right\|_{\mathcal{H}^{\prime}}^{p^{\prime}} \mathrm{d} t & \leq \int_{0}^{T}\left|\left\langle\frac{\partial u^{(n)}}{\partial t}, \psi\right\rangle\right|^{p^{\prime}} \mathrm{d} t \\
\leq & \left.\left.C \int_{0}^{T}\left|\sum_{k=1}^{n} \chi_{k}(t) \int_{\Omega}\right| \Delta u_{k}\right|^{p-2} \Delta u_{k} \Delta \psi \mathrm{d} x\right|^{p^{\prime}} \mathrm{d} t \\
& +\left.\left.C \int_{0}^{T}\left|\sum_{k=1}^{n} \chi_{k}(t) \int_{\Omega} f(x)\right| \nabla u_{k}\right|^{q-2} \nabla u_{k} \nabla \psi \mathrm{d} x\right|^{p^{\prime}} \mathrm{d} t
\end{aligned}
$$




$$
\begin{aligned}
& +C \int_{0}^{T}\left|\sum_{k=1}^{n} \chi_{k}(t) \int_{\Omega} g(x) \psi \mathrm{d} x\right|^{p^{\prime}} \mathrm{d} t \\
\leq & C\left(\left\|\Delta w^{(n)}\right\|_{L^{p}\left(Q_{T}\right)}^{p}+\left\|\nabla w^{(n)}\right\|_{L^{p}\left(Q_{T}\right)}^{p}+1\right) \\
\leq & C
\end{aligned}
$$

for any $\psi \in \mathcal{H}$ with $\|\psi\|_{\mathcal{H}} \leq 1$. It yields the first estimate $\left\|\frac{\partial u^{(n)}}{\partial t}\right\|_{L^{p^{\prime}\left(0, T ; \mathcal{H}^{\prime}\right)}} \leq$ C.

By (9), it follows that

$$
\begin{aligned}
& \left\|u^{(n)}\right\|_{L^{\alpha}\left(0, T ; L^{2}(\Omega)\right)}^{\alpha} \leq \int_{0}^{T}\left(\int_{\Omega}\left|u^{(n)}\right|^{2} \mathrm{~d} x\right)^{\frac{\alpha}{2}} \mathrm{~d} t \\
= & C^{\alpha} \int_{0}^{T}\left(\int_{\Omega}\left|\sum_{k=1}^{n} \chi_{k}(t)\left[\lambda_{k}(t) u_{k}(x)+\left(1-\lambda_{k}(t)\right) u_{k-1}(x)\right]\right|^{2} \mathrm{~d} x\right)^{\frac{\alpha}{2}} \mathrm{~d} t \\
= & C^{\alpha} \sum_{k=1}^{n} \int_{(k-1) h}^{(k h)}\left(\int_{\Omega}\left|\left[\lambda_{k}(t) u_{k}(x)+\left(1-\lambda_{k}(t)\right) u_{k-1}(x)\right]\right|^{2} \mathrm{~d} x\right)^{\frac{\alpha}{2}} \mathrm{~d} t \\
\leq & C^{\alpha} \sum_{k=1}^{n} h\left(\int_{\Omega}\left(\left|u_{k}(x)\right|^{2}+\left|u_{k-1}(x)\right|^{2}\right) \mathrm{d} x\right)^{\frac{\alpha}{2}} \\
\leq & C^{\alpha}
\end{aligned}
$$

where the constant $C>0$ is independent of $\alpha>1$, and thus

$$
\left\|u^{(n)}\right\|_{L^{\infty}\left(0, T ; L^{2}(\Omega)\right)}=\lim _{\alpha \rightarrow \infty}\left\|u^{(n)}\right\|_{L^{\alpha}\left(0, T ; L^{2}(\Omega)\right)} \leq C .
$$

Theorem 1.1 is proved as follows.

Proof of Theorem 1.1. By Lemma 2.2, there exist a subsequence of $w^{(n)}$, a function $v \in L^{p^{\prime}}\left(Q_{T}\right)$ and a vector function $v_{2} \in\left(L^{p^{\prime}}\left(Q_{T}\right)\right)^{N}$ such that

$$
\begin{aligned}
& w^{(n)} \stackrel{*}{\rightarrow} u \text { weakly* in } L^{\infty}\left(0, T ; L^{2}(\Omega)\right), \\
& w^{(n)} \rightarrow u \text { weakly in } L^{p}\left(0, T ; W^{2, p}(\Omega)\right), \\
& \left|\Delta w^{(n)}\right|^{p-2} \Delta w^{(n)} \rightarrow v_{1} \text { weakly in } L^{p^{\prime}}\left(Q_{T}\right), \\
& \left|\nabla w^{(n)}\right|^{p-2} \nabla w^{(n)} \rightarrow v_{2} \text { weakly in }\left(L^{p^{\prime}}\left(Q_{T}\right)\right)^{N}
\end{aligned}
$$

when $n \rightarrow \infty$. Lemma 2.3 yields

$$
\begin{aligned}
& \frac{\partial u^{(n)}}{\partial t} \rightarrow \frac{\partial \rho}{\partial t} \text { weakly in } L^{p^{\prime}}\left(0, T ; \mathcal{H}^{\prime}\right), \\
& u^{(n)} \stackrel{*}{\rightarrow} \rho \text { weakly* in } L^{\infty}\left(0, T ; L^{2}(\Omega)\right) .
\end{aligned}
$$

According to $(7)$, for any test function $\varphi \in C_{0}^{\infty}\left(Q_{T}\right)$, we have

$$
\left|\iint_{Q_{T}}\left(w^{(n)}-u^{(n)}\right) \varphi \mathrm{d} x \mathrm{~d} t\right|
$$




$$
\begin{aligned}
& =\left|\iint_{Q_{T}} \sum_{k=1}^{n} \chi_{k}(t)\left(1-\lambda_{k}(t)\right)\left(u_{k}-u_{k-1}\right) \varphi \mathrm{d} x \mathrm{~d} t\right| \\
& \leq \sum_{k=1}^{n} \int_{0}^{T} \chi_{k}(t) h\left(\left.\left|\int_{\Omega}\right| \Delta u_{k}\right|^{p-2} \Delta u_{k} \Delta \varphi \mathrm{d} x|+| \int_{\Omega} f\left|\nabla u_{k}\right|^{q-2} \nabla u_{k} \nabla \varphi \mathrm{d} x \mid \mathrm{d} t\right. \\
& \left.\quad+\left|\int_{\Omega} h \varphi \mathrm{d} x\right|\right) \mathrm{d} t \\
& \leq h \int_{0}^{T} \quad\left(\left.\left|\int_{\Omega}\right| \Delta w^{(n)}\right|^{p-2} \Delta w^{(n)} \Delta \varphi \mathrm{d} x|+| \int_{\Omega} f\left|\nabla w^{(n)}\right|^{q-2} \nabla w^{(n)} \nabla \varphi \mathrm{d} x \mid\right. \\
& \left.\quad+\left|\int_{\Omega} h \varphi \mathrm{d} x\right|\right) \mathrm{d} t \\
& \leq C h \rightarrow 0,
\end{aligned}
$$

when $n \rightarrow \infty$. It gives $\rho=u$ a.e. in $Q_{T}$. Now we can perform the limit $n \rightarrow \infty$ in the following equality:

$$
\begin{aligned}
& \int_{0}^{T}\left\langle\frac{\partial u^{(n)}}{\partial t}, \varphi\right\rangle \mathrm{d} t+\iint_{Q_{T}}\left|\Delta w^{(n)}\right|^{p-2} \Delta w^{(n)} \Delta \varphi \mathrm{d} x \mathrm{~d} t \\
& +\iint_{Q_{T}} f(x)\left|\nabla w^{(n)}\right|^{q-2} \nabla w^{(n)} \nabla \varphi \mathrm{d} x \mathrm{~d} t=\iint_{Q_{T}} g(x) \varphi \mathrm{d} x \mathrm{~d} t
\end{aligned}
$$

for any test function $\varphi$. Hence, by $n \rightarrow \infty$, we get

$$
\begin{aligned}
& \int_{0}^{T}\left\langle\frac{\partial u}{\partial t}, \varphi\right\rangle \mathrm{d} t+\iint_{Q_{T}} v_{1} \Delta \varphi \mathrm{d} x \mathrm{~d} t+\iint_{Q_{T}} f(x) v_{2} \nabla \varphi \mathrm{d} x \mathrm{~d} t \\
= & \iint_{Q_{T}} g(x) \varphi \mathrm{d} x \mathrm{~d} t .
\end{aligned}
$$

It is necessary to prove that $v_{1}=|\Delta u|^{p-2} \Delta u$ and $v_{2}=|\nabla u|^{q-2} \nabla u$. Taking $u$ as a test function in (14), we have

$$
\begin{aligned}
& \frac{1}{2} \int_{\Omega}|u(x, T)|^{2} \mathrm{~d} x-\frac{1}{2} \int_{\Omega}\left|u_{0}\right|^{2} \mathrm{~d} x+\iint_{Q_{T}} v_{1} \Delta u \mathrm{~d} x \mathrm{~d} t \\
& +\iint_{Q_{T}} f(x) v_{2} \nabla u \mathrm{~d} x \mathrm{~d} t=\iint_{Q_{T}} g(x) u \mathrm{~d} x \mathrm{~d} t .
\end{aligned}
$$

In addition, by choosing $\phi=w^{(n)}$ in (7), we obtain

$$
\begin{aligned}
& \frac{1}{2} \int_{\Omega}\left|w^{(n)}(x, T)\right|^{2} \mathrm{~d} x-\frac{1}{2} \int_{\Omega}\left|u_{0}\right|^{2} \mathrm{~d} x+\iint_{Q_{T}}\left|\Delta w^{(n)}\right|^{p} \mathrm{~d} x \mathrm{~d} t \\
& +\iint_{Q_{T}} f(x)\left|\nabla w^{(n)}\right|^{p} \mathrm{~d} x \mathrm{~d} t \\
\leq & \iint_{Q_{T}} g(x) w^{(n)} \mathrm{d} x \mathrm{~d} t .
\end{aligned}
$$


Notice that for any test functions $\varphi_{1}, \varphi_{2}$ and any constant $\varepsilon>0$,

$$
\begin{aligned}
& \iint_{Q_{T}}\left(\left|\zeta_{1}^{(n)}\right|^{p-2} \zeta_{1}^{(n)}-\left|\eta_{1 \varepsilon}\right|^{p-2} \eta_{1 \varepsilon}\right)\left(\zeta_{1}^{(n)}-\eta_{1 \varepsilon}\right) \mathrm{d} x \mathrm{~d} t \geq 0, \\
& \iint_{Q_{T}}\left(\left|\zeta_{2}^{(n)}\right|^{p-2} \zeta_{2}^{(n)}-\left|\eta_{2 \varepsilon}\right|^{p-2} \eta_{2 \varepsilon}\right)\left(\zeta_{2}^{(n)}-\eta_{2 \varepsilon}\right) \mathrm{d} x \mathrm{~d} t \geq 0,
\end{aligned}
$$

where $\left.\zeta_{1}^{(n)}=\Delta w^{(n)}, \eta_{1 \varepsilon}=\Delta\left(u-\varepsilon \varphi_{1}\right)\right), \zeta_{2}^{(n)}=\nabla w^{(n)}$ and $\left.\eta_{2 \varepsilon}=\nabla\left(u-\varepsilon \varphi_{2}\right)\right)$. In view of (16)-(18), by letting $n \rightarrow \infty$, we have

$$
\begin{aligned}
& \frac{1}{2} \int_{\Omega}|u(x, T)|^{2} \mathrm{~d} x-\frac{1}{2} \int_{\Omega}\left|u_{0}\right|^{2} \mathrm{~d} x-\iint_{Q_{T}}\left|\Delta\left(u-\varepsilon \varphi_{1}\right)\right|^{p} \mathrm{~d} x \mathrm{~d} t \\
& +\iint_{Q_{T}}\left|\Delta\left(u-\varepsilon \varphi_{1}\right)\right|^{p-2} \Delta\left(u-\varepsilon \varphi_{1}\right) \Delta u \mathrm{~d} x \mathrm{~d} t+\iint_{Q_{T}} v_{1} \Delta\left(u-\varepsilon \varphi_{1}\right) \mathrm{d} x \mathrm{~d} t \\
& \quad-\iint_{Q_{T}} f(x)\left|u-\varepsilon \varphi_{2}\right|^{p} \mathrm{~d} x \mathrm{~d} t+\iint_{Q_{T}} f(x) v_{2} \nabla\left(u-\varepsilon \varphi_{2}\right) \mathrm{d} x \mathrm{~d} t \\
& +\iint_{Q_{T}} f(x)\left|\nabla\left(u-\varepsilon \varphi_{2}\right)\right|^{p-2} \nabla\left(u-\varepsilon \varphi_{2}\right) \nabla u \mathrm{~d} x \mathrm{~d} t \\
& \leq \iint_{Q_{T}} g(x) u \mathrm{~d} x \mathrm{~d} t .
\end{aligned}
$$

Employ (15) to obtain

$$
\begin{aligned}
& \iint_{Q_{T}}\left[\left|\Delta\left(u-\varepsilon \varphi_{1}\right)\right|^{p-2} \Delta\left(u-\varepsilon \varphi_{1}\right)-v_{1}\right] \Delta \varphi_{1} \mathrm{~d} x \mathrm{~d} t \\
& +\iint_{Q_{T}} f(x)\left[\left|\nabla\left(u-\varepsilon \varphi_{2}\right)\right|^{p-2} \nabla\left(u-\varepsilon \varphi_{2}\right)-v_{2}\right] \nabla \varphi_{2} \mathrm{~d} x \mathrm{~d} t \leq 0 .
\end{aligned}
$$

By taking $\varepsilon \rightarrow 0$, one has

$$
\iint_{Q_{T}}\left[|\Delta u|^{p-2} \Delta u-v_{1}\right] \Delta \varphi_{1} \mathrm{~d} x \mathrm{~d} t+\iint_{Q_{T}} f(x)\left[|\nabla u|^{p-2} \nabla u-v_{2}\right] \nabla \varphi_{2} \mathrm{~d} x \mathrm{~d} t \leq 0 .
$$

Taking $\varphi_{2}=0$ and applying the arbitrariness of $\varphi_{1}$, we conclude that $|\Delta u|^{p-2} \Delta u=v_{1}$ a.e. in $Q_{T}$. By defining $v_{2}=|\nabla u|^{p-2} \nabla u$ in $\left(Q_{T}^{1}\right)^{N} \doteq(\{x \in$ $\Omega \mid f(x)=0\} \times(0, T))^{N}$, we get $v_{2}=|\nabla u|^{p-2} \nabla u$ a.e. in $\left(Q_{T}\right)^{N}$. Then (14) becomes

$$
\begin{aligned}
& \int_{0}^{T}\left\langle\frac{\partial u}{\partial t}, \varphi\right\rangle \mathrm{d} t+\iint_{Q_{T}}|\Delta u|^{p-2} \Delta u \Delta \varphi \mathrm{d} x \mathrm{~d} t \\
& +\iint_{Q_{T}} f(x)|\nabla u|^{p-2} \nabla u \nabla \varphi \mathrm{d} x \mathrm{~d} t=\iint_{Q_{T}} g(x) \varphi \mathrm{d} x \mathrm{~d} t .
\end{aligned}
$$

A standard argument can show that $u \in C\left([0, T] ; L^{p}(\Omega)\right)$ from

$$
u_{t} \in L^{p^{\prime}}\left(0, T ; \mathcal{H}^{\prime}\right) \text { and } u \in L^{p}\left(0, T ; W^{2, p}(\Omega)\right)
$$

(see $[9])$.

Similar to the proof of Lemma 2.1, we can prove the uniqueness. 


\section{Long time behavior}

In this section, we give the proof of the long-time behavior of solutions when $h \equiv 0$.

Proof of Theorem 1.2. We define an entropy functional as following

$$
H(t) \doteq \frac{1}{2} \int_{\Omega} u^{2} \mathrm{~d} x
$$

It is easy to check that

$$
\frac{\mathrm{d} H(t)}{\mathrm{d} t}=-\int_{\Omega}|\Delta u|^{p} \mathrm{~d} x-\int_{\Omega} f(x)|\nabla u|^{q} \mathrm{~d} x .
$$

Combining Poincaré inequality with Sobolev imbedding theorem, we get

$$
\frac{\mathrm{d} H(t)}{\mathrm{d} t} \leq-C\left(\int_{\Omega}|u|^{2} \mathrm{~d} x\right)^{\frac{p}{2}}
$$

When $p \geq 2$, by solving the differential equation $y^{\prime}=-C y^{\frac{p}{2}}$, we have

$$
\|u\|_{L^{2}(\Omega)}(t) \leq \begin{cases}\left(\left\|u_{0}\right\|_{L^{2}(\Omega)}^{2-p}+C\left(\frac{p}{2}-1\right) t\right)^{-\frac{1}{p-2}}, & p>2 \\ \left\|u_{0}\right\|_{L^{2}(\Omega)} e^{-C t}, & p=2\end{cases}
$$

On the other hand, for $p<2$, one has

$$
\|u\|_{L^{2}(\Omega)}^{2-q} \leq\left\|u_{0}\right\|_{L^{2}(\Omega)}^{2-q}-C\left(1-\frac{p}{2}\right) t
$$

where the constant $C$ is independent of $t$. Therefore, there exists a time $\bar{t}$ such that $\|u\|_{L^{2}(\Omega)} \leq 0$ for all $t>\bar{t}$. It yields the assertion of Theorem 1.2.

\section{References}

[1] R. A. Adams, Sobolev Space, Academic Press, New York, 1975.

[2] F. Bernis and A. Friedman, Higher order nonlinear degenerate parabolic equations, J. Differential Equations 83 (1990), no. 1, 179-206.

[3] J. M. Cahn and J. E. Hilliard, Free energy of a non-uniform system I. Interfacial free energy, J. Chem. Phys. 28 (1958), 258-367.

[4] J. A. Carrillo and G. Toscani, Long-time asymptotics for strong solutions of the thin film equation, Commu. Math. Phys. 225 (2002), no. 3, 551-571.

[5] D. Gilbarg and N. S. Trudinger, Elliptic Partial Different Equations of Second Order, Second ed., Springer-Verlag, New York, 1983.

[6] G. Grün, Degenerate parabolic differential equations of fourth order and a plasticity model with nonlocal hardening, Z. Anal. Anwendungen 14 (1995), no. 3, 541-574.

[7] B. Liang and S. Zheng, Existence and asymptotic behavior of solutions to a nonlinear parabolic equation of fourth-order, J. Math. Anal. Appl. 348 (2008), no. 1, 234-243.

[8] T. G. Myers, Thin films with high surface tension, SIAM Rev. 40 (1998), no. 3, 441-462.

[9] J. Simon, Compact sets in the space $L^{p}(0, T ; B)$, Ann. Math. Pura. Appl. 146 (1987), 65-96.

[10] M. Xu and S. Zhou, Existence and uniqueness of weak solutions for a generalized thin film equation, Nonlinear Anal. 60 (2005), no. 4, 755-774.

[11] Stability and regularity of weak solutions for a generalized thin film equation, J. Math. Anal. Appl 337 (2008), no. 1, 49-60. 
BO LIANG

School of ScIENCE

Dalian JiaOtong University

Dalian 116028, P. R. China

E-mail address: cnliangbo@163.com

Meishan WANG

SCHOOL OF SCIENCE

Dalian JiaOtong University

DALiAn 116028, P. R. ChinA

E-mail address: wangmeishanTina@126.com

YING WANG

School of ScIEnce

Dalian JiaOtong University

Dalian 116028, P. R. China

E-mail address: wangyingfc@163.com 